This is an electronic reprint of the original article. This reprint may differ from the original in pagination and typographic detail.

Author(s): Kuznetsov, Nikolay; Leonov, G. A.; Yuldashev, M. V.; Yuldashev, R. V.

Title: $\quad$ Hidden attractors in dynamical models of phase-locked loop circuits : limitations of simulation in MATLAB and SPICE

Year: $\quad 2017$

Version:

Please cite the original version:

Kuznetsov, N., Leonov, G. A., Yuldashev, M. V., \& Yuldashev, R. V. (2017). Hidden attractors in dynamical models of phase-locked loop circuits : limitations of simulation in MATLAB and SPICE. Communications in Nonlinear Science and Numerical Simulation, 51, 39-49. https://doi.org/10.1016/j.cnsns.2017.03.010

All material supplied via JYX is protected by copyright and other intellectual property rights, and duplication or sale of all or part of any of the repository collections is not permitted, except that material may be duplicated by you for your research use or educational purposes in electronic or print form. You must obtain permission for any other use. Electronic or print copies may not be offered, whether for sale or otherwise to anyone who is not an authorised user. 


\section{Accepted Manuscript}

Hidden attractors in dynamical models of phase-locked loop circuits: limitations of simulation in MATLAB and SPICE

Kuznetsov N. V., Leonov G. A., Yuldashev M. V., Yuldashev R. V.

PII:

DOI:

Reference:

$$
\text { S1007-5704(17)30090-4 }
$$$$
\text { 10.1016/j.cnsns.2017.03.010 }
$$

CNSNS 4137
Communications in

Nonlinear Science and

Numerical Simulation

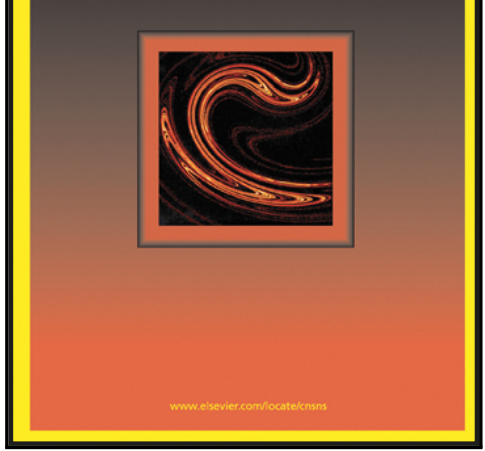

To appear in: $\quad$ Communications in Nonlinear Science and Numerical Simulation

Received date: $\quad 30$ August 2016

Revised date: $\quad 4$ November 2016

Accepted date: $\quad 13$ March 2017

Please cite this article as: Kuznetsov N. V., Leonov G. A., Yuldashev M. V., Yuldashev R. V., Hidden attractors in dynamical models of phase-locked loop circuits: limitations of simulation in MATLAB and SPICE, Communications in Nonlinear Science and Numerical Simulation (2017), doi: 10.1016/j.cnsns.2017.03.010

This is a PDF file of an unedited manuscript that has been accepted for publication. As a service to our customers we are providing this early version of the manuscript. The manuscript will undergo copyediting, typesetting, and review of the resulting proof before it is published in its final form. Please note that during the production process errors may be discovered which could affect the content, and all legal disclaimers that apply to the journal pertain. 


\title{
Hidden attractors in dynamical models of phase-locked loop circuits: limitations of simulation in MATLAB and SPICE.
}

\author{
Kuznetsov N. V.a,b,c,*, Leonov G. A. ${ }^{\mathrm{b}, \mathrm{d}}$, Yuldashev M. V. ${ }^{\mathrm{b}}$, Yuldashev R. V. ${ }^{\mathrm{b}}$ \\ ${ }^{a}$ Modeling Evolutionary Algorithms Simulation and Artificial Intelligence, Faculty of Electrical 8 Electronics Engineering, Ton Duc \\ Thang University, Ho Chi Minh, Vietnam \\ ${ }^{b}$ Faculty of Mathematics and Mechanics, Saint-Petersburg State University, Russia \\ ${ }^{c}$ Dept. of Mathematical Information Technology, University of Jyväskylä, Finland \\ ${ }^{d}$ Institute of Problems of Mechanical Engineering RAS, Russia
}

\begin{abstract}
During recent years it has been shown that hidden oscillations, whose basin of attraction does not overlap with small neighborhoods of equilibria, may significantly complicate simulation of dynamical models, lead to umreliable results and wrong conclusions, and cause serious damage in drilling systems, aircrafts control systems, electromechanical systems, and other applications. In this article a survey of various phase-locked loop based circuits (used in satellite navigation systems, optical, and digital communication), where such difficulties take place in MATLAB and SPICE, is provided. Considered examples can be used for testing other phase-locked loop based circuits and simulation tools, and motivate the development and application of rigorous analytical methods for the global analysis of phase-locked loop based circuits.
\end{abstract}

Keywords: Phase-locked loop, two-phase PLL, optical Costas loop, simulation, MATLAB, SPICE, synchronization, nonlinear control system, dynamical system, hidden attractor, multistability

\section{Introduction}

The phase-locked loop (PLL) based circuits are widely used nowadays in various applications such as telecommunications, computer architectures, navigation (e.g. in GPS, GLONASS) and many others. One of the main features of PLL-based circuits is synchronization of the controlled oscillator (slave oscillator) frequency to the frequency of reference signal (master oscillator). An important engineering characteristic of PLL-based circuit is a set of frequency deviations for which the PLL-based circuit achieves a synchronized (locked) state for any initial state $[1,2]$ : for a dynamical model of PLL-based circuit in the signal's phase space the pull-in range corresponds to such frequency deviations that any solution of dynamical model is attracted to one of the equilibria (rigorous definition can be found in $[3-5])$.

Since PLL is essentially nonlinear control system and its nonlinear analysis is a challenging task (see, e.g. [4, 612]), in practice, simulation is widely used for the study of PLL-based circuits (for a discussion of PLL-based circuits simulation in SPICE and MATLAB see, e.g. [13-15]). However, recently it was shown that simulation may not reveal complex behavior of PLL: such examples where the simulation of PLL-based circuits leads to unreliable results, are demonstrated in $[16,17]$. These examples demonstrate the difficulties of numerical search of so-called hidden oscillations, whose basin of attraction does not overlap with the neighborhood of an equilibrium point, and thus may be difficult to find numerically [18-20]. In this case the observation of one or another stable solution may depend on the initial data and integration step.

This article provides a survey of various classical PLL-based circuits for which difficulties of simulation related to the hidden oscillations take place in MATLAB and SPICE. Considered examples can be used for testing other simulation software and models of PLL-based circuits. S. Goldman, who has worked at Texas Instruments over 20 years, notes [21, p.XIII] that PLLs are used as pipe cleaners for breaking simulation tools. Also the considered examples motivate to develop and apply rigorous analytical methods for the global analysis of PLL-based circuits $[4]$.

\section{Simulation of PLL-based circuits}

\subsection{Simulation of two-phase phase-locked loop}

Let us consider the two-phase PLL operation $([22,23])$ (see Fig. 1). The input carrier is $\cos \left(\theta_{\text {ref }}(t)\right)$ with

\footnotetext{
*Corresponding author email: nikolayv.kuznetsov@tdt.edu.vn, nkuznetsov239@gmail.com
} 


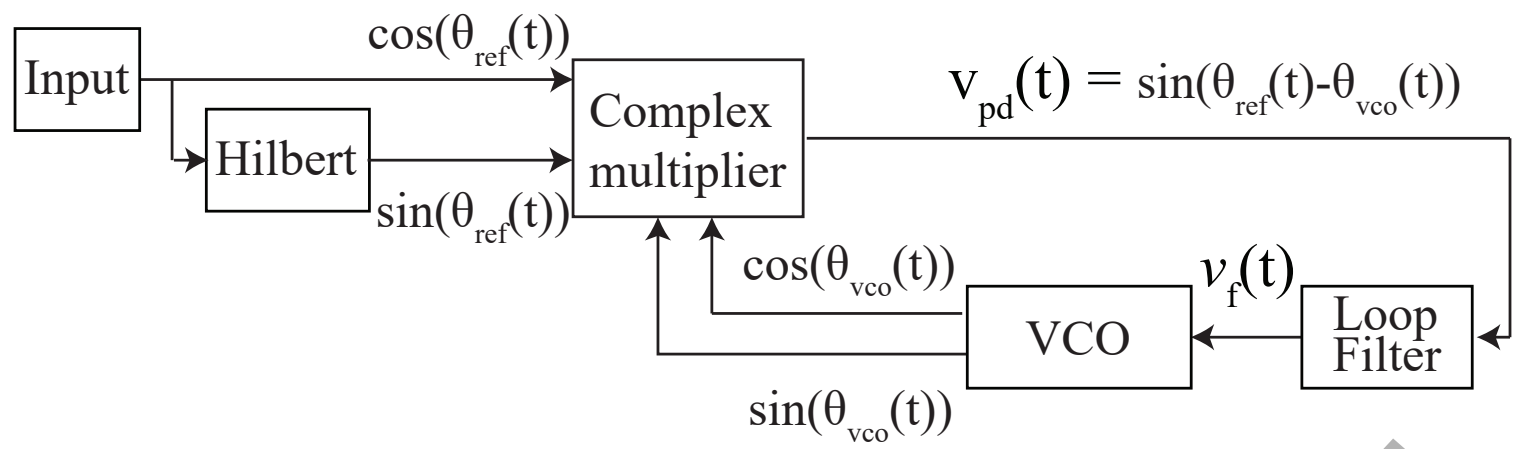

Figure 1: Two-phase PLL model in the signal space

$\theta_{\text {ref }}(t)$ as a phase. Hilbert block shifts the phase of the carrier by $90^{\circ}$ producing the output $\sin \left(\theta_{\text {ref }}(t)\right)$. The voltage-controlled oscillator (VCO) generates $\sin \left(\theta_{\mathrm{vco}}(t)\right)$ and $\cos \left(\theta_{\mathrm{vco}}(t)\right)$. Figure 2 shows the structure of complex multiplier (phase detector). The phase detector consists of two analog multipliers and analog subtractor. The

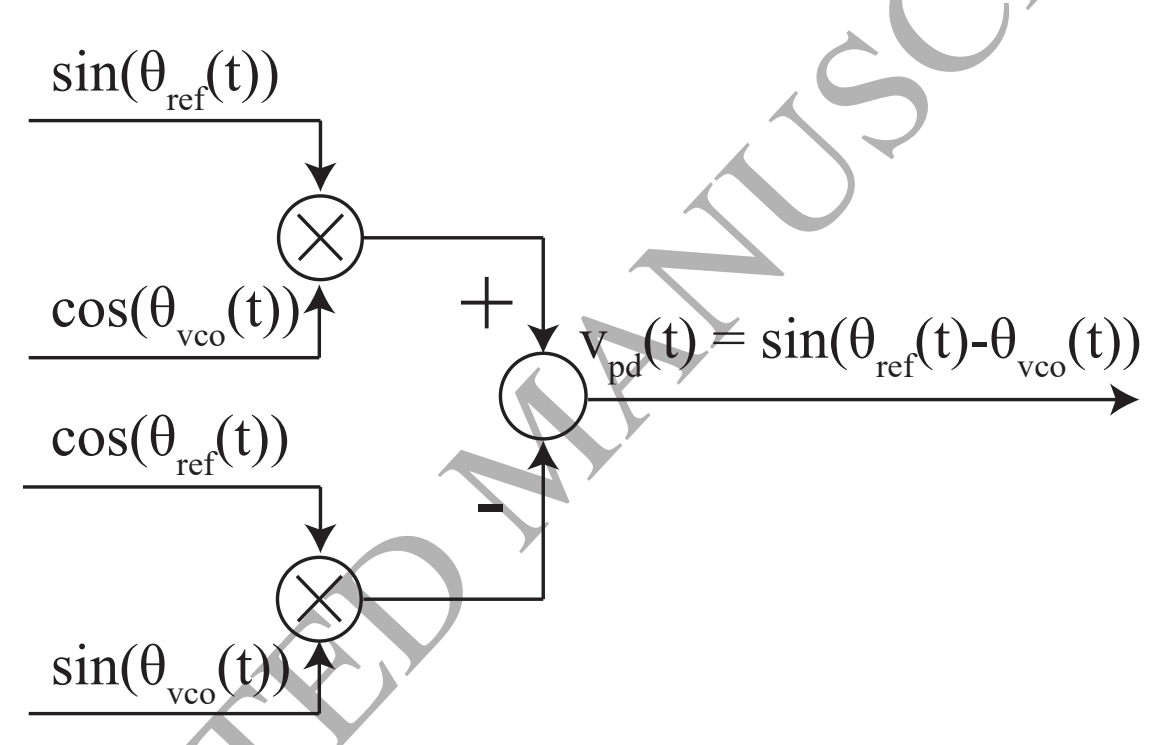

Figure 2: Phase detector (complex multiplier) in the two-phase PLL

output of phase detector is as follows

$$
v_{\mathrm{pd}}(t)=\sin \left(\theta_{\text {ref }}(t)-\theta_{\mathrm{vco}}(t)\right) .
$$

The relation between the input $v_{\mathrm{pd}}(t)$ and the output $v_{f}(t)$ of the Loop filter has the following form

$$
\dot{x}=A x+b v_{\mathrm{pd}}(t), \quad v_{f}(t)=c^{*} x+h v_{\mathrm{pd}}(t),
$$

where $A$ is a constant $(n \times n)$-matrix, $x \in \mathbb{R}^{n}$ is the Loop filter state, $b$ and $c$ are constant vectors, and $h$ is a number. A widely used lead-lag filter is described by the transfer function

$$
\begin{aligned}
& H(s)=c^{*}(s I-A)^{-1} b+h=\frac{a(s)}{d(s)}=\frac{1+\tau_{2} s}{1+\left(\tau_{1}+\tau_{2}\right) s}, \\
& A=-\frac{1}{\tau_{1}+\tau_{2}}, \quad b=1-\frac{\tau_{2}}{\tau_{1}+\tau_{2}}, \quad c=\frac{1}{\tau_{1}+\tau_{2}}, \quad h=\frac{\tau_{2}}{\tau_{1}+\tau_{2}},
\end{aligned}
$$

where $\tau_{1}>0$ and $\tau_{2}>0$ are parameters. The error signal $v_{f}(t)$ adjusts the VCO frequency to the frequency of the input signal:

$$
\dot{\theta}_{\mathrm{vco}}=\omega_{\mathrm{vco}}^{\text {free }}+K_{\mathrm{vco}} v_{f}(t)
$$




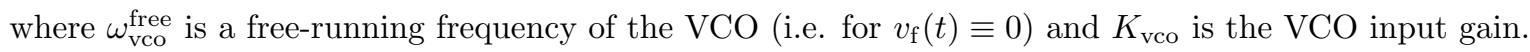

In contrast to the classical PLL-based circuits this model does not contain high-frequency components in the output of phase detector (complex multiplier). Therefore the two-phase PLL model in the signal space is equivalent to the model in the signal's phase space (Fig. 3). This model considers only phases $\theta_{\text {vco }}$ and $\theta_{\text {ref }}$, and, thus, simplifies analytical and numerical study. The frequency of the input signal (reference frequency) is usually assumed to be

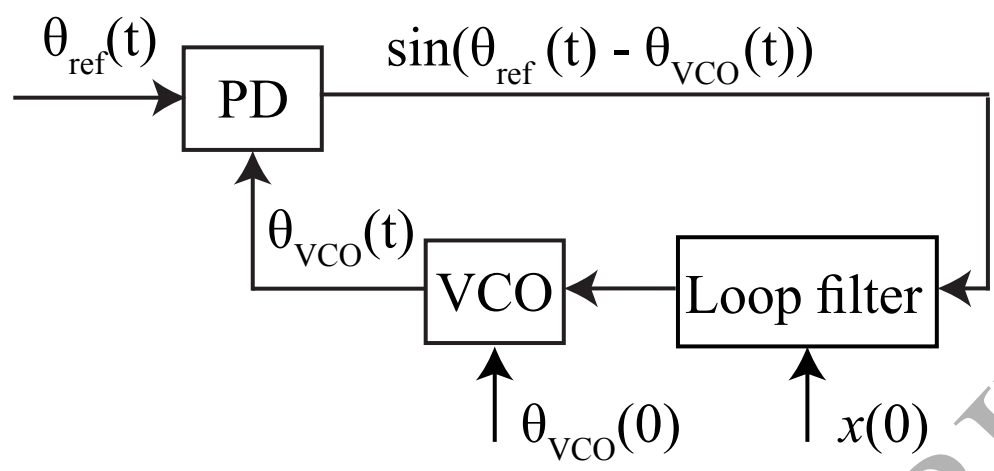

Figure 3: Two-phase PLL model in the signal's phase space

constant:

$$
\dot{\theta}_{\text {ref }}(t) \equiv \omega_{\text {ref }}
$$

Introduce

$$
\theta_{e}(t)=\theta_{\text {ref }}(t)-\theta_{\text {vco }}(t), \quad \omega_{e}^{\text {free }}=\omega_{\text {ref }}-\omega_{\text {yco }}^{\text {free }}
$$

where $\left|\omega_{e}^{\text {free }}\right|$ is called a frequency deviation. Combining equations (1),(2),(3), and (4) we get the following equations of the model in the signal's phase space

$$
\begin{aligned}
& \dot{x}=\frac{-1}{\tau_{1}+\tau_{2}} x+\left(1-\frac{\tau_{2}}{\tau_{1}+\tau_{2}}\right) \sin \left(\theta_{e}\right), \\
& \dot{\theta}_{e}=\omega_{e}^{\text {free }}-K_{\text {veo }}\left(\frac{1}{\tau_{1}+\tau_{2}} x+\frac{\tau_{2}}{\tau_{1}+\tau_{2}} \sin \left(\theta_{e}\right)\right) .
\end{aligned}
$$

The equilibria points of (6) are defined as follows

$$
x_{e q}=\tau_{1} \sin \left(\theta_{e q}\right), \sin \left(\theta_{e q}\right)=\frac{\omega_{e}^{\text {free }}}{K_{\text {vco }}}
$$

and their local analysis can be done by the Routh-Hurwitz criterion. To study the global stability and the pull-in range we apply numerical methods.

Consider MATLAB Simulink model of the two-phase PLL (Fig. 4). We use the block Loop filter to take into account the initial filter state $x(0)$; the initial phase error $\theta_{e}(0)$ can be taken into account by the property initial data of the Intergator blocks.

The simulation results of the Loop filter output are shown in Fig. 5. If the max step size parameter is "auto", then the simulation shows that the two-phase PLL synchronizes to the carrier (left subfigure). This fact suggests that $\omega_{e}^{\text {free }}$ belongs to the pull-in range. However, more precise simulation with the max step size set to "1e-4" shows that the Loop filter output does not synchronize to the carrier (right subfigure). Considered oscillations shows that $\omega_{e}^{\text {free }}$ can not belong to the pull-in range.

Now we consider the corresponding simulation in SPICE. Consider the two-phase PLL model in SPICE (Fig. 6). This model corresponds to Fig. 1 and Fig. 2. The model in Fig. 6 corresponds to the following NGSPICE listing ${ }^{1}$ :

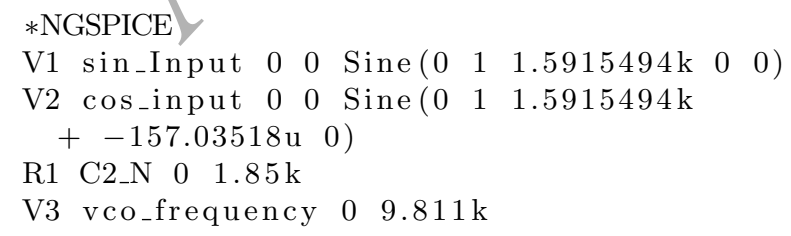

\footnotetext{
${ }^{1}$ Official ngspice web page http://ngspice.sourceforge.net/. Online realization is available at http://www.ngspice.com/index. php?public_circuit=AK3QmA
} 


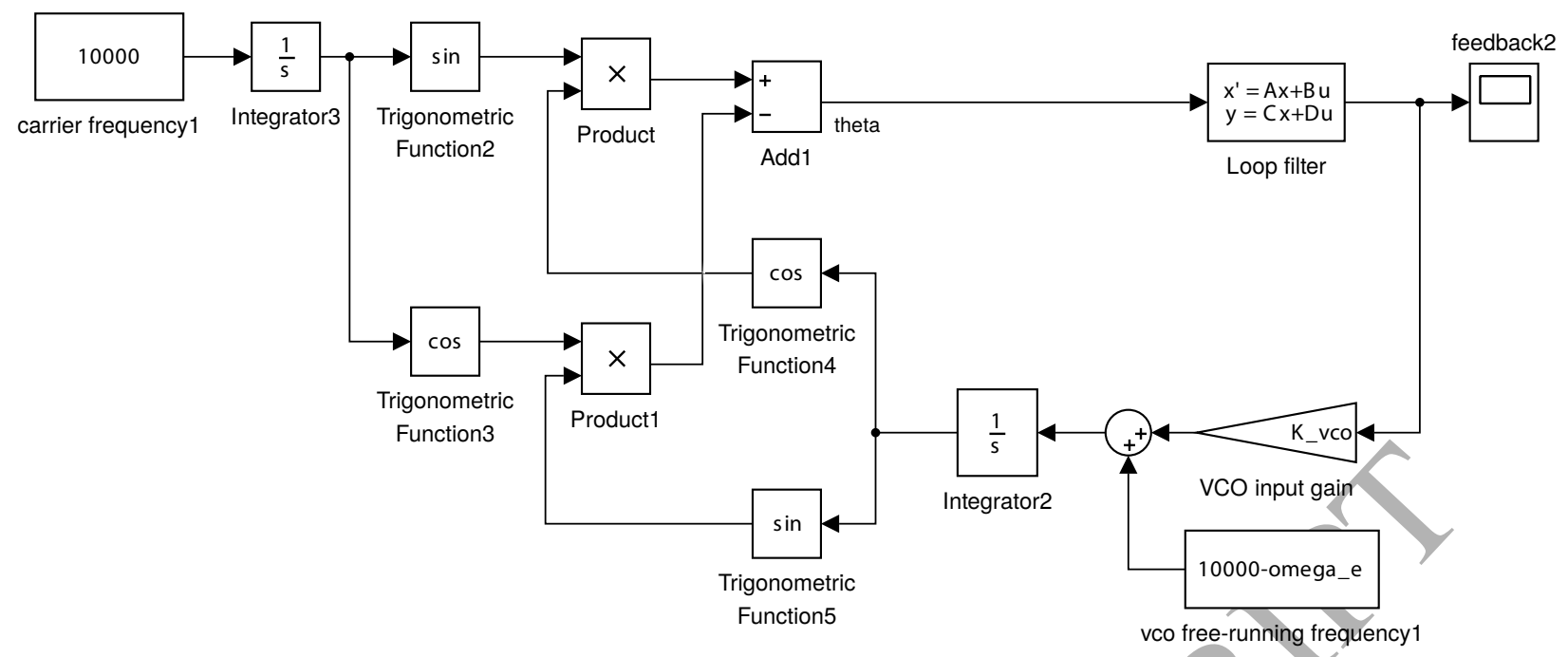

Figure 4: Realization of the two-phase PLL in MATLAB Simulink $\left(A \rightarrow \mathrm{A}, b \rightarrow \mathrm{B}, c \rightarrow \mathrm{C}, h \rightarrow \mathrm{D} ; \omega_{e}^{\mathrm{free}} \rightarrow\right.$ omega_e, $K_{\mathrm{vco}} \rightarrow \mathrm{K} \_$vco, $\left.\omega_{\text {ref }}=10000\right)$.

7 R2 filter_out PD_output $4.48 \mathrm{k}$ subckt multiplier1 N1 N2 OUT B1 OUT $0 \quad \mathrm{~V}=\mathrm{V}(\mathrm{N} 1) * \mathrm{~V}(\mathrm{~N} 2)$

.ends multiplier 1

Xmult1 sin_Input vco_cos_output ARB1_OUT

+ multiplier 1 $*$

. subckt multiplier 2 N1 N2 OUT

B1 OUT $0 \quad \mathrm{~V}=\mathrm{V}(\mathrm{N} 1) * \mathrm{~V}(\mathrm{~N} 2)$

.ends multiplier 2

Xmult2 cos_input vco_sin_output E3_CN + multiplier 2

Aintegrator N1 OUT time_count

. model time_count int ( in_offset $=0.0$ gain $=1.0$

+ out_lower_limit $=-1 \mathrm{e} 12$

+ out_upper_limit $=1$ e 12

+ limit_range $=1 \mathrm{e}-9$ out_ic $=0.0$ )

. ends integrator

Xintegrator integrator_in integrator_out

+ integrator

E2 integrator_in 0 vco_frequency E2_CN 1

C2 filter_out C2_N $10 u \quad I C=50 \mathrm{~m}$

E3 PD_output 0 ARB1_OUT E3_CN 1

E6 E2_CN 0 filter_out $0-250$

* .TRAN $1 \mathrm{~m} 5$ 50 1m UIC 

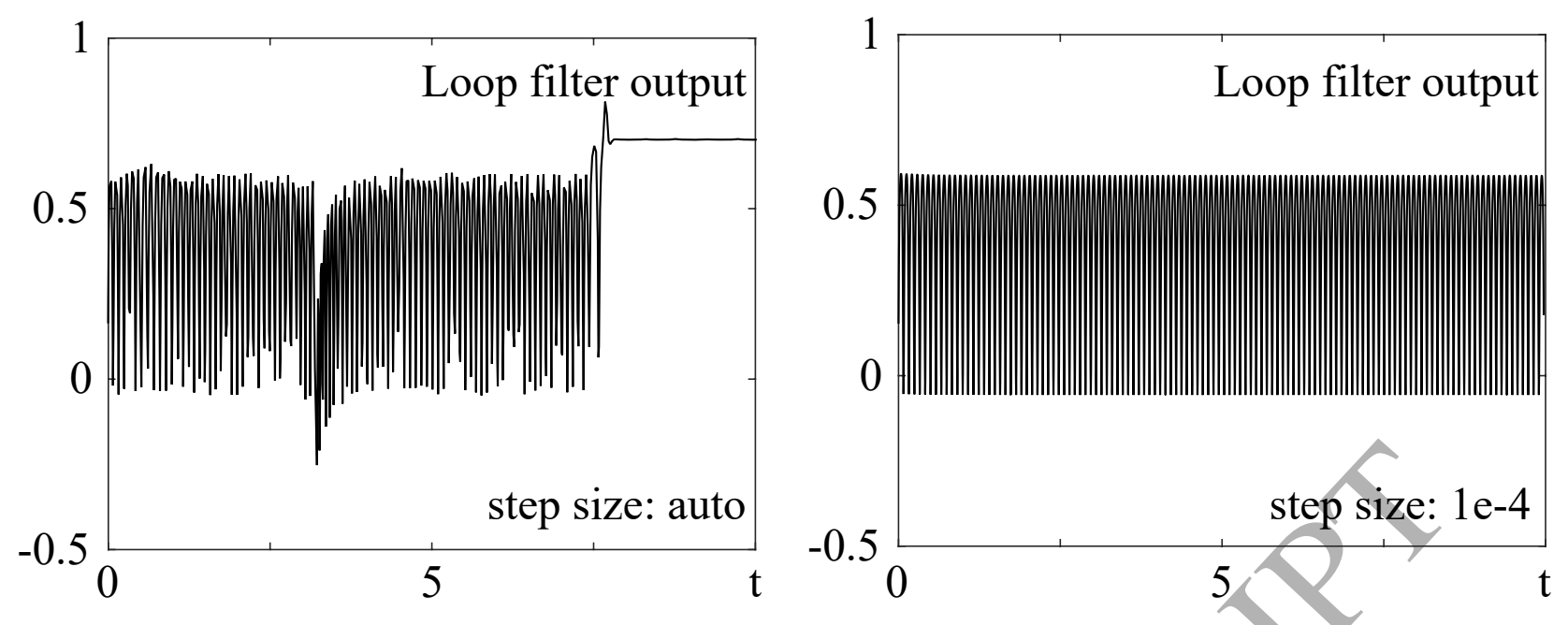

Figure 5: Simulation of the two-phase PLL in MATLAB. Simulation of the same circuit shows qualitatively different results for different simulation steps (sampling, integration step). The left subfigure shows synchronization (step size "auto"), the right subfigure oscillations (step size "1e-4"). Parameters: $K$-vco $=250, \mathrm{~A}=-15.7978, \mathrm{~B}=0.7077, \mathrm{C}=15.7978, \mathrm{D}=0.2923\left(\tau_{1}=0.0448, \tau_{2}=\right.$ $0.0185)$, omega_e $=178.9$, initial state of the Loop filter is $x(0)=0.01, \theta_{e}(0)=0$.

\section{.TRAN $10 \mathrm{~m} \quad 5 \quad 0 \quad 10 \mathrm{~m}$ UIC}

The results of simulation are shown in Fig. 7. If the integration step in SPICE is equal to $10 m$, then the simulation shows that VCO locks to the reference signal. The simulation with step $1 \mathrm{~m}$ reveals oscillations. To consider the performance effect of additive white Gaussian noise (AWGN) added to the input signal one can use the following code-snippet for $\mathrm{NGSPICE}^{2}$ :

E16 sin_noise 0 sin_Input noise_1 1

E17 cos_noise 0 cos_input noise_2 1

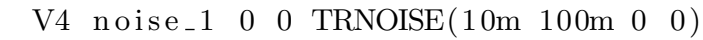

4 V5 noise_2 00 TRNOISE $(10 \mathrm{~m} \quad 100 \mathrm{~m} \quad 0 \quad 0)$

In this example the two-phase PLL may lock or not lock depending on noise. Also to make the model more realistic one may consider non-linear VCO characteristic (see, e.g. example of hidden oscillations with non-linear VCO in [24]), the effect of frequency dependence of Hilbert transformer, and the effect of asymmetry of complex multiplier. However, for simplicity in this article we follow the classical analysis of the pull-in range $[1,2]$.

\subsection{Simulation of the optical Costas loop}

Consider the optical Costas loop model (see, e.g. [25]). The input signal is a BPSK (Binary Phase Shift Keying) signal, which is the product of the transferred data $m(t)= \pm 1$ and the harmonic carrier $\sqrt{P_{\text {ref }}} \sin \left(\theta_{\text {ref }}(t)\right)$ with high frequency $\omega_{\text {ref }}(t)=\dot{\theta}_{\text {ref }}(t)$. The VCO signal is sinusoidal $\sqrt{P_{\mathrm{vco}}} \sin \left(\theta_{\mathrm{vco}}(t)\right)$ with the frequency $\omega_{\mathrm{vco}}(t)=\dot{\theta}_{\mathrm{vco}}(t)$. Block $90^{\circ}$ Hybrid has the following outputs:

$$
\begin{aligned}
E_{1} & =\frac{1}{2}\left(m(t) \sqrt{P_{\text {ref }}} \cos \left(\theta_{\mathrm{ref}}(t)\right)+\sqrt{P_{\mathrm{vco}}} \cos \left(\theta_{\mathrm{vco}}(t)\right)\right), \\
E_{2} & =\frac{1}{2}\left(m(t) \sqrt{P_{\text {ref }}} \cos \left(\theta_{\mathrm{ref}}(t)\right)-\sqrt{P_{\mathrm{vco}}} \cos \left(\theta_{\mathrm{vco}}(t)\right)\right), \\
E_{3} & =\frac{1}{2}\left(m(t) \sqrt{P_{\text {ref }}} \cos \left(\theta_{\mathrm{ref}}(t)\right)+\sqrt{P_{\mathrm{vco}}} \cos \left(\theta_{\mathrm{vco}}(t)+\frac{\pi}{2}\right)\right), \\
E_{4} & =\frac{1}{2}\left(m(t) \sqrt{P_{\mathrm{ref}}} \cos \left(\theta_{\mathrm{ref}}(t)\right)-\sqrt{P_{\mathrm{vco}}} \cos \left(\theta_{\mathrm{vco}}(t)+\frac{\pi}{2}\right)\right) .
\end{aligned}
$$

\footnotetext{
${ }^{2}$ Official ngspice web page http://ngspice.sourceforge.net/. Online realization is available at http://www.ngspice.com/index. php?public $\backslash_{-}$circuit $=A v 4 \mathrm{Mbb}$
} 


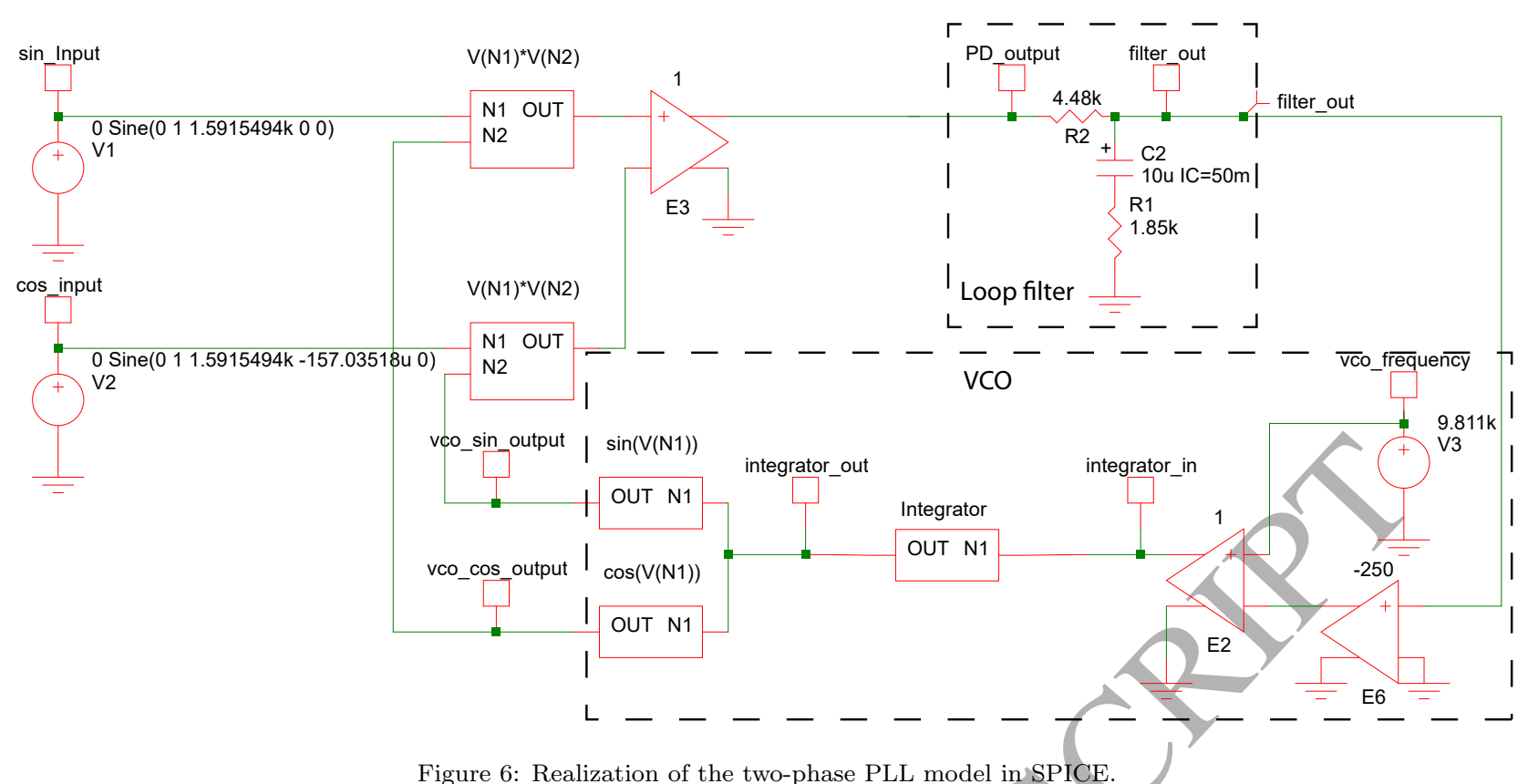

Figure 6: Realization of the two-phase PLL model in SPICE.
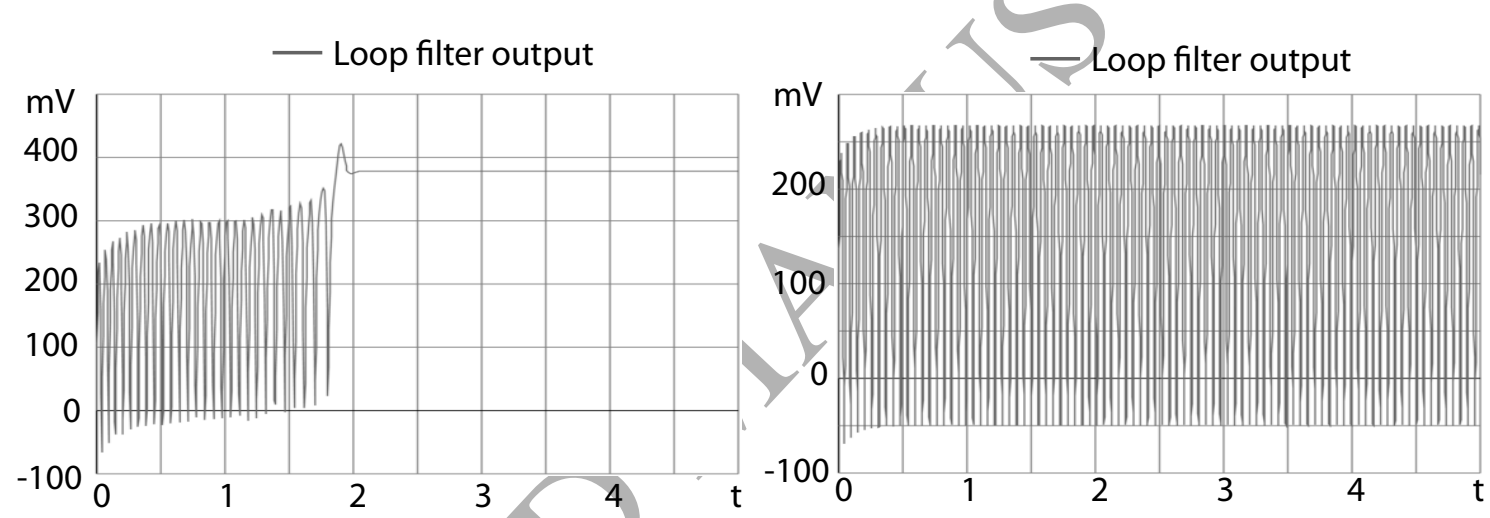

Figure 7: Simulation of the two-phase PLL in NGSPICE. For default simulation step (10m) the VCO synchronizes to the reference signal (left subfigure). For simulation step $1 \mathrm{~m}$ an undamped oscillation exists (right subfigure). Parameters: $K_{\mathrm{vco}}=250, \mathrm{~A}=-15.7978$, $\mathrm{b}=0.7077, \mathrm{c}=15.7978, \mathrm{~h}=0.2923\left(\tau_{1}=0.0448, \tau_{2}=0.0185\right), \omega_{e}^{\text {free }}=189$. Initial charge of capacitor $C 2$ is $50 \mathrm{~m}$ (corresponds to initial state of the Loop filter $), \theta_{e}(0)=0, \omega_{\text {ref }}=10000$.

Four outputs of the receivers are the following

$$
\begin{aligned}
I_{1}(t) & =\frac{R}{8}\left(P_{\text {ref }}+P_{\mathrm{vco}}+2 m(t) \sqrt{P_{\text {ref }} P_{\mathrm{vco}}} \cos \left(\theta_{e}(t)\right)\right), \\
I_{2}(t) & =\frac{R}{8}\left(P_{\text {ref }}+P_{\mathrm{vco}}-2 m(t) \sqrt{P_{\text {ref }} P_{\mathrm{vco}}} \cos \left(\theta_{e}(t)\right)\right), \\
I_{3}(t) & =\frac{R}{8}\left(P_{\text {ref }}+P_{\mathrm{vco}}+2 m(t) \sqrt{P_{\text {ref }} P_{\mathrm{vco}}} \cos \left(\theta_{e}(t)-\frac{\pi}{2}\right)\right), \\
I_{4}(t) & =\frac{R}{8}\left(P_{\text {ref }}+P_{\mathrm{vco}}-2 m(t) \sqrt{P_{\text {ref }} P_{\mathrm{vco}}} \cos \left(\theta_{e}(t)-\frac{\pi}{2}\right)\right) .
\end{aligned}
$$

The amplifier multiplies its inputs by a positive number $a$, and then two pairs of signals are subtracted:

$$
\begin{aligned}
& I_{I}(t)=a I_{1}(t)-a I_{2}(t)=\frac{m(t) R a \sqrt{P_{\mathrm{ref}} P_{\mathrm{vco}}}}{2} \cos \left(\theta_{e}(t)\right), \\
& I_{Q}(t)=a I_{3}(t)-a I_{4}(t)=\frac{m(t) R a \sqrt{P_{\mathrm{ref}} P_{\mathrm{vco}}}}{2} \cos \left(\theta_{e}(t)-\frac{\pi}{2}\right) .
\end{aligned}
$$




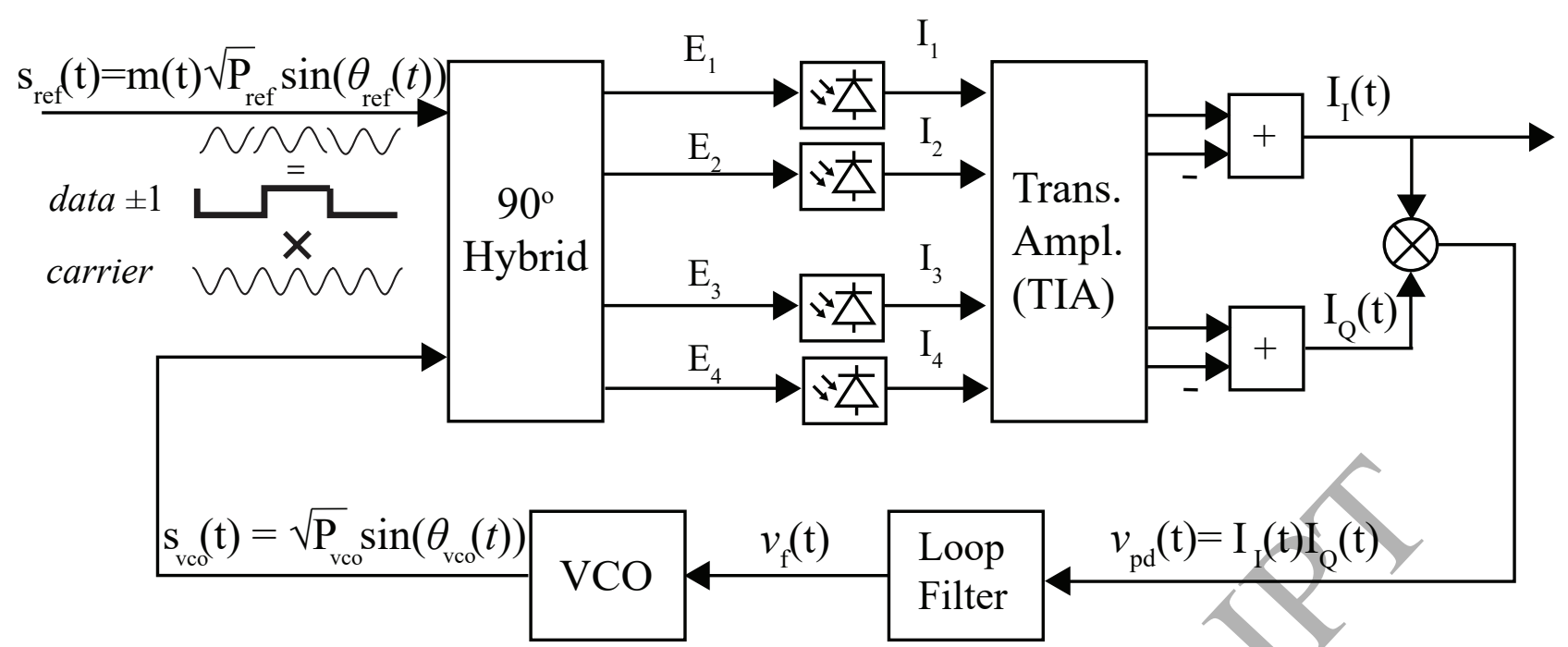

Figure 8: The optical Costas loop in the signal space

After the multiplication $\otimes$ the Loop filter input becomes

$$
v_{\mathrm{pd}}(t)=I_{I}(t) I_{Q}(t)=\frac{R^{2} a^{2} P_{\mathrm{ref}} P_{\mathrm{vco}}}{4} \cos \left(\theta_{e}(t)\right) \sin \left(\theta_{e}(t)\right)=\frac{R^{2} a^{2} P_{\mathrm{ref}} P_{\mathrm{vco}}}{8} \sin \left(2 \theta_{e}(t)\right)
$$

Combining equations (11), (2), (3), and (4) we get nonlinear model of optical Costas loop. This model corresponds to the classical signal's phase model of PLL shown in Fig. 3. Now we construct the corresponding model in MATLAB Simulink (see Fig. 9).

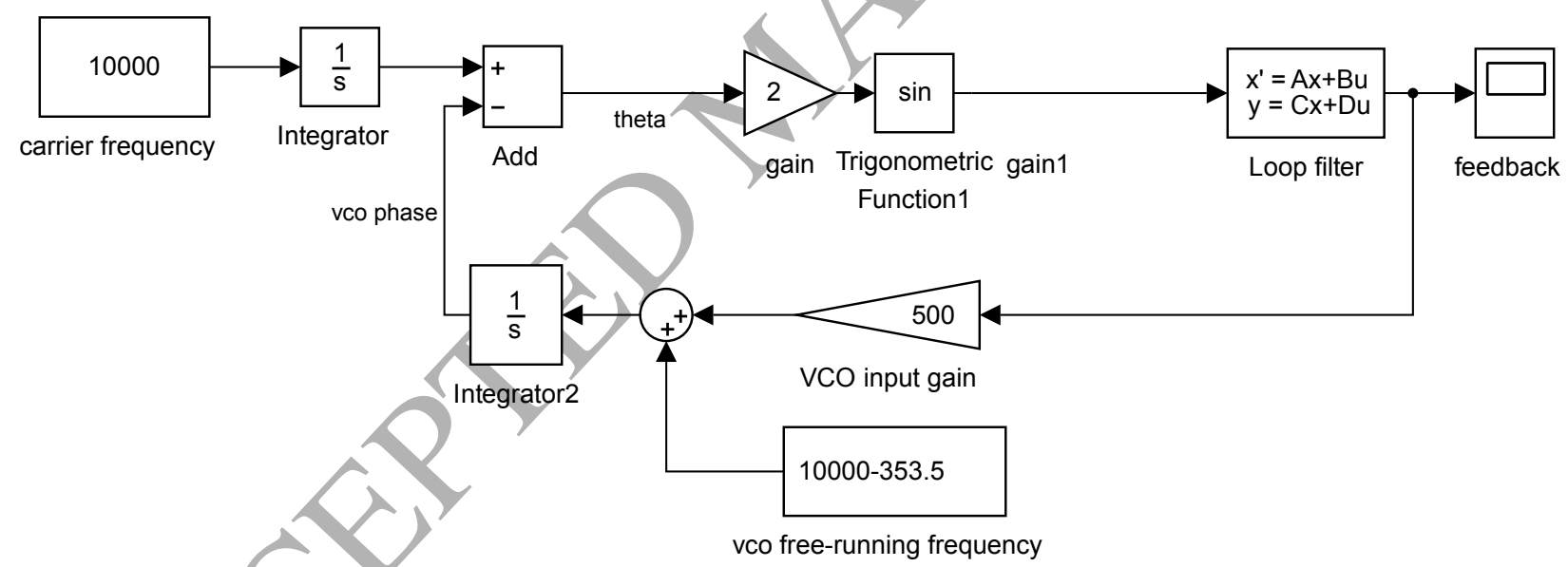

Figure 9: Signal's phase space model of optical Costas loop in MATLAB Simulink $\left(A \rightarrow \mathrm{A}, b \rightarrow \mathrm{B}, c \rightarrow \mathrm{C}, h \rightarrow \mathrm{D} ; \omega_{e}^{\text {free }}=353.5\right.$, $\left.K_{\mathrm{vco}}=500, \omega_{\text {ref }}=10000\right)$.

The simulation of optical Costas loop reveals the same effect, as in previous examples. 

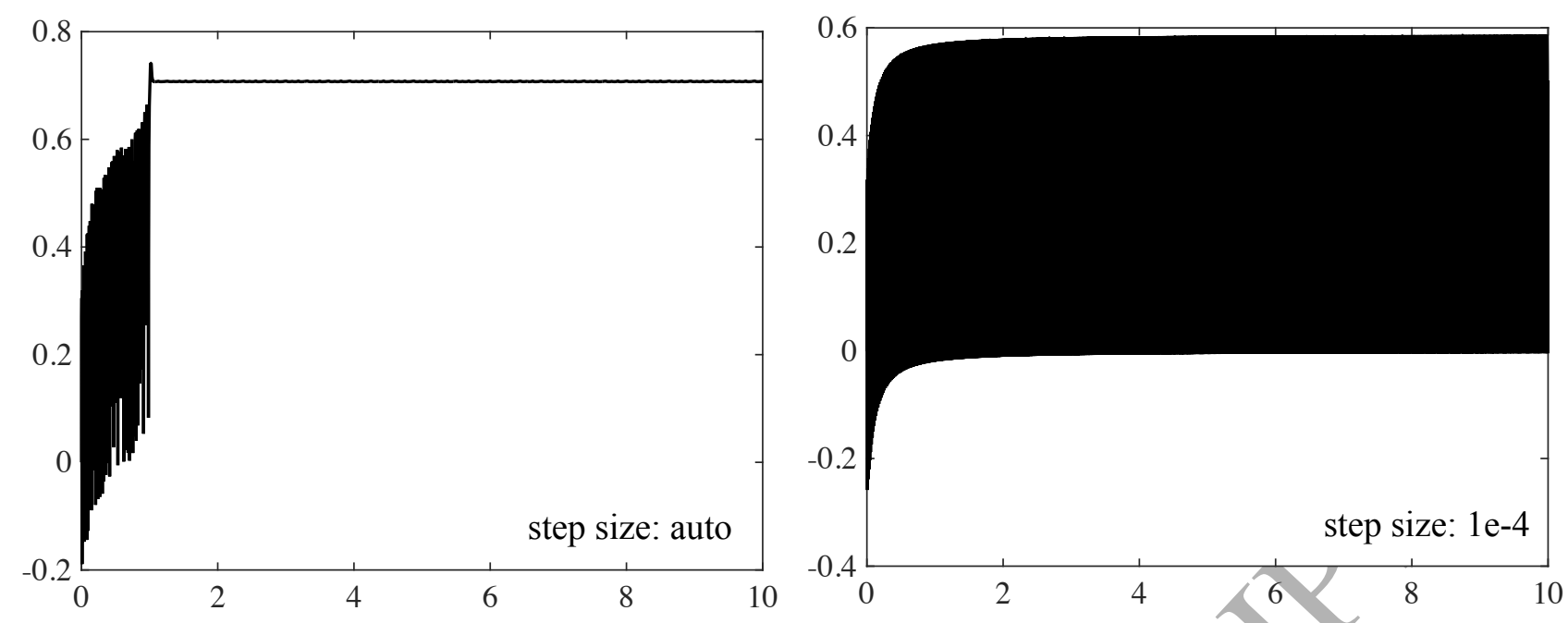

Figure 10: Simulation of the optical Costas loop in MATLAB Simulink (Fig. 9), Loop filter output. The left subfigure shows synchronization (step size "auto"), the right subfigure — oscillations (step size "1e-4"). Parameters: VCO input gain is $K_{\mathrm{vco}}=500$, $A=-15.7978, b=0.7077, c=15.7978, h=0.2923$. Initial state of Loop filter is zero, $\theta_{e}(0)=0$.

If the max step size parameter is "auto", then simulation shows that the model of optical Costas loop synchronizes to the carrier (left subfigure) and, thus, the considered $\omega_{e}^{\text {free }}$ can correpond to the pull-in range. However, if the max step size is set to "1e-4", then the Loop filter output does not synchronize to the carrier (right subfigure) and, thus, the considered $\omega_{e}^{\text {free }}$ is outside of the pull-in range.

\subsection{Simulation of the BPSK Costas loop}

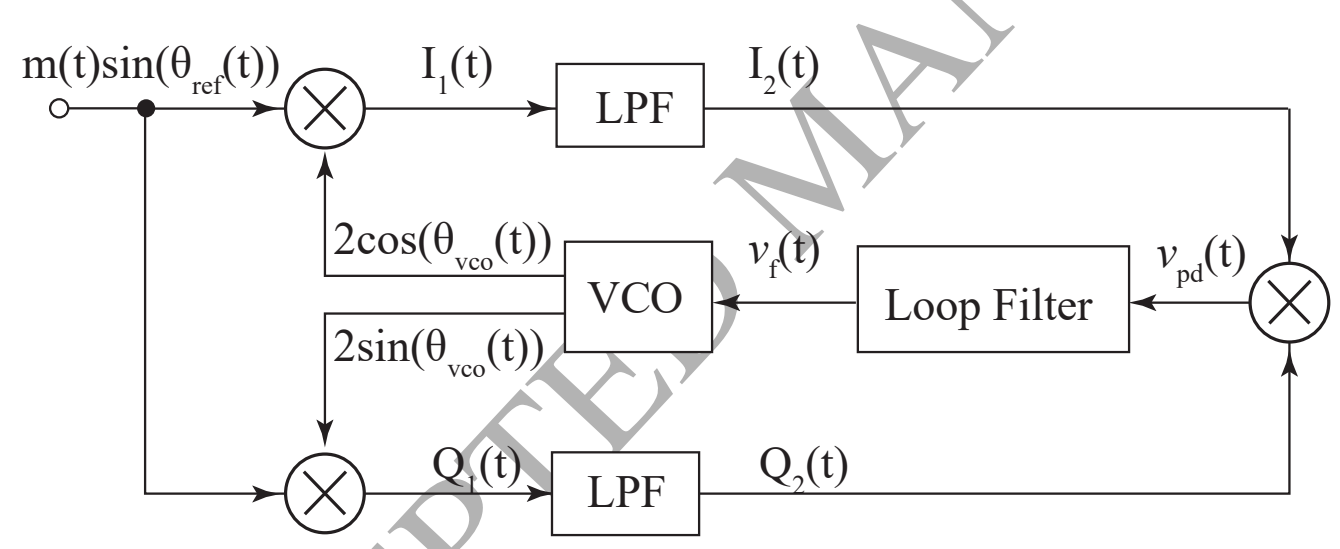

Figure 11: BPSK Costas loop

Consider the BPSK Costas loop circuit [5, 26, 27], which is used for carrier recovery and signal demodulation (Fig. 11). The input signal is a Binary Phase-Shift Keying (BPSK) signal, which is the product of data $m(t)$ and carrier $\sin \left(\theta_{\text {ref }}(t)\right)$. In the following analysis we are not interested in $m(t)$, therefore we consider $m(t) \equiv 1$. The input signal is multiplied by the VCO outputs $2 \cos \left(\theta_{\mathrm{vco}}(t)\right)$ and $2 \sin \left(\theta_{\mathrm{vco}}(t)\right)$ :

$$
\begin{aligned}
& I_{1}(t)=2 \cos \left(\theta_{\mathrm{vco}}(t)\right) \sin \left(\theta_{\text {ref }}(t)\right), \\
& Q_{1}(t)=2 \sin \left(\theta_{\mathrm{vco}}(t)\right) \sin \left(\theta_{\text {ref }}(t)\right) .
\end{aligned}
$$

The transfer functions of low-pass filters LPF are equal to $H(s)=\frac{1}{\frac{s}{a}+1}$ (i.e. $\left.A=-a, b=a, c=1, h=0\right)$. The product of outputs of low-pass filters $v_{\mathrm{pd}}(t)=I_{2}(t) Q_{2}(t)$ is filtered by the Loop filter defined by $(3)$. The VCO phase is defined by (4). The frequency of the input signal (reference frequency) is usually assumed to be constant, i.e.

$$
\theta_{\text {ref }}(t)=\omega_{\text {ref }} t
$$




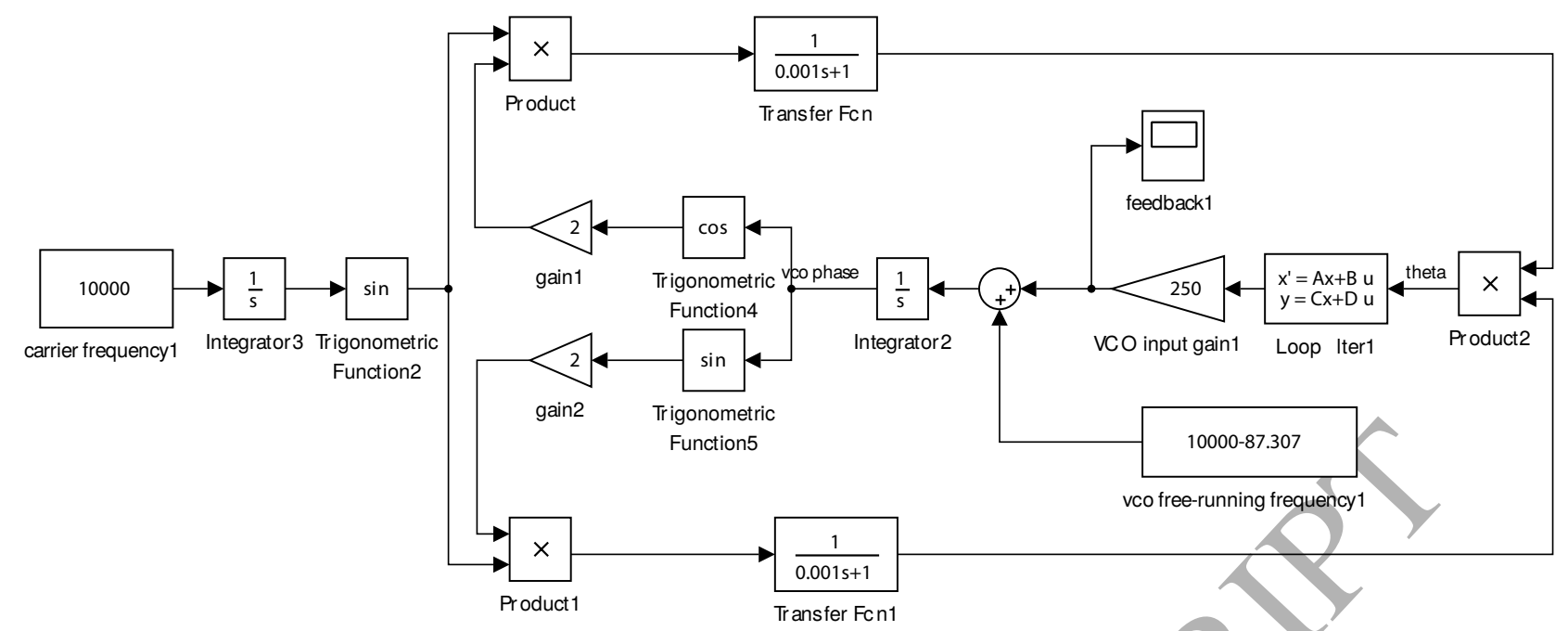

Figure 12: MATLAB Simulink model of BPSK Costas loop in the signal space $\left(A \rightarrow \mathrm{A}, b \rightarrow \mathrm{B}, \mathrm{c} \rightarrow \mathrm{C}, h \rightarrow \mathrm{D} ; \omega_{e}^{\text {free }}=87.307\right.$, VCO input gain is $K_{\mathrm{vco}}=250, \omega_{\text {ref }}=10000, a=1000$ )

Therefore the BPSK Costas loop is described by the following equations

$$
\begin{aligned}
& \dot{x}_{1}=-a x_{1}+2 a \sin \left(\omega_{\mathrm{ref}} t\right) \cos \left(\theta_{\mathrm{vco}}(t)\right), \\
& \dot{x}_{2}=-a x_{2}+2 a \sin \left(\omega_{\mathrm{ref}} t\right) \sin \left(\theta_{\mathrm{vco}}(t)\right), \\
& \dot{x}=\frac{-1}{\tau_{1}+\tau_{2}} x+\left(1-\frac{\tau_{2}}{\tau_{1}+\tau_{2}}\right) x_{1} x_{2}, \\
& \dot{\theta}_{\mathrm{vco}}=\omega_{\mathrm{vco}}^{\text {free }}+K_{\mathrm{vco}}\left(\frac{1}{\tau_{1}+\tau_{2}} x+\frac{\tau_{2}}{\tau_{1}+\tau_{2}} x_{1} x_{2}\right) .
\end{aligned}
$$

The model of BPSK Costas loop in MATLAB is shown in Fig. 12. Here blocks "LPF" are modeled using Transfer Function element from the standard library.

In Fig. 13 the BPSK Costas loop model, simulated with relative tolerance set to $10^{-5}$ or smaller, does not acquire lock. However the model, simulated with standard parameters (the max step size set to "auto"), acquires lock after approximately 12 seconds. Here the input signal frequency is 10000, the VCO free-running frequency $\omega_{e}^{\text {free }}=87.307$, the VCO input gain is $K_{\mathrm{vco}}=250$, the initial state of the Loop filter is $x(0)=0$.
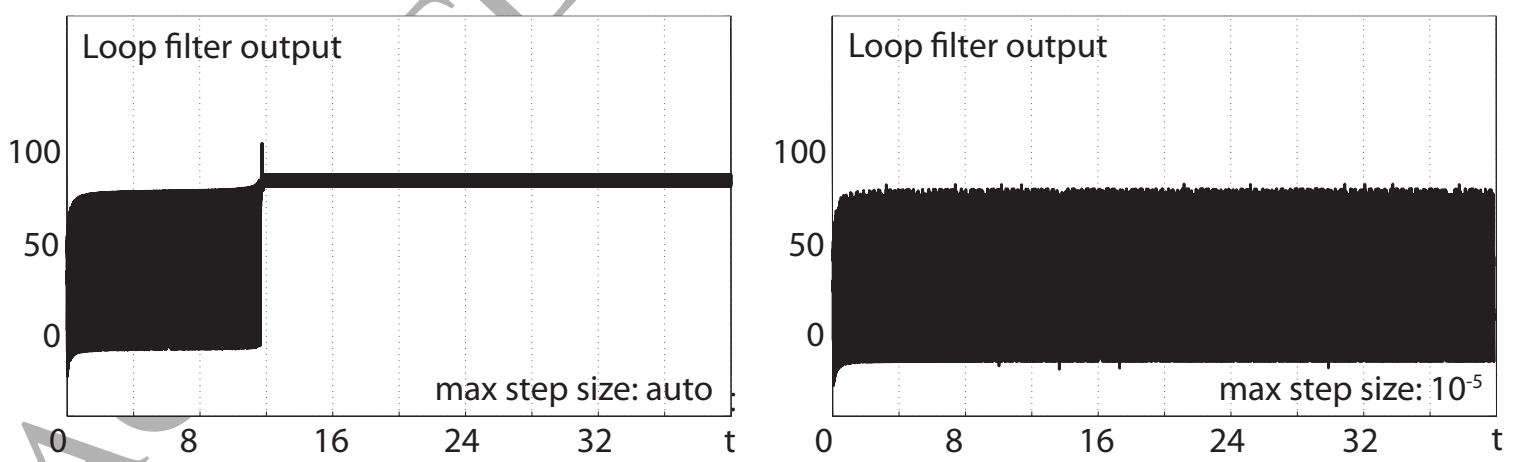

Figure 13: Simulation of the BPSK Costas loop in MATLAB. The Loop filter outputs $v_{f}(t)$ for the initial data $x_{0}=0, \theta_{e}(0)=0$, obtained for default "auto" max step size and max step size set to $10^{-5}$. Parameters: $A=-15.7978, b=0.7077, c=15.7978, h=0.2923$ $\left(\tau_{1}=0.0448, \tau_{2}=0.0185\right)$, VCO input gain is $K_{\mathrm{vco}}=250, a=1000, \omega_{e}^{\text {free }}=87.307$.

Note that any initial state of the Loop filter less than 0.4 leads to a similar effect.

\section{Hidden oscillations}

Now we give mathematical explanation of the considered problems of simulation. An oscillation in a dynamical system can be localized numerically if the initial conditions from its open neighborhood lead to the long-time 
behavior that approaches the oscillation. Such an oscillation (or a set of oscillations) is called an attractor, and its attracting set is called a basin of attraction. Thus, from a point of view of the numerical analysis of nonlinear dynamical models it is essential to classify an attractor as self-excited or hidden attractor depending on simplicity of finding its basin of attraction [18-20]: An attractor is called a self-excited attractor if its basin of attraction intersects with an arbitrarily small open neighborhood of an unstable equilibrium, otherwise it is called a hidden attractor.

For a self-excited attractor, its basin of attraction is connected with an unstable equilibrium and, thus, it can be localized numerically by a standard computational procedure in which after a transient process a trajectory, starting from a point in a neighborhood of the unstable equilibrium, reaches a state of oscillation and, therefore, visualizes the attractor. While many classical attractors are self-exited attractors and therefore can be obtained numerically by the standard computational procedure, for localization of hidden attractors it is necessary to develop special analytical-numerical procedures in which initial point is chosen from the basin of attraction. The numerical search of hidden attractors can be also complicated due to the small size of the basin of attraction with respect to the considered set of parameters and subset of the phase space (see, e.g. a discussion of rare hidden attractors in $[28,29])$. For example, hidden attractors are attractors in the system without equilibria or in multistable system with only stable equilibria. During recent years it has been shown that hidden attractors may/significantly complicate simulation of dynamical models, lead to unreliable results and wrong conclusions, and even cause serious damage in aircrafts control systems [30, 31], drilling systems [32-34], electromechanical systems [35], and other applications $[36,37]$. Recent examples of hidden attractors can be found e.g. in [29, 35, 38-45].

Following pioneering works $[1,2]$, classical PLL-based circuits are often described by a models in the signal's phase space (rigorous justification can be done by the averaging methods, see, e.g. the corresponding discussion in [46-49]). One of the first analytical study of classical PLL circuit with lead-lag Loop filter in the signal's phase space was done in 1956 [50] by the phase-plane analysis. In this work Kapranov assumed that all oscillations in the considered two-dimensional model are self-excited. However, in 1961 Gubar' [51] revealed a gap in Kapranov's consideration and showed that in classical PLL circuit with lead-lag, Loop filter a stable periodic trajectory, which is a hidden attractor, can exist and bound the basin of attraction of equilibria. This stable periodic trajectory (stable cycle) coexists with an unstable periodic trajectory (unstable cycle) and if the gap between these two trajectories is small (see Fig. 14a) and the discretization step (sampling) in numerical integration is larger than this gap, then the numerical integration may step over both stable and unstable periodic trajectories. We can avoid this problem if we choose simulation step much smaller than the distance between the cycles.

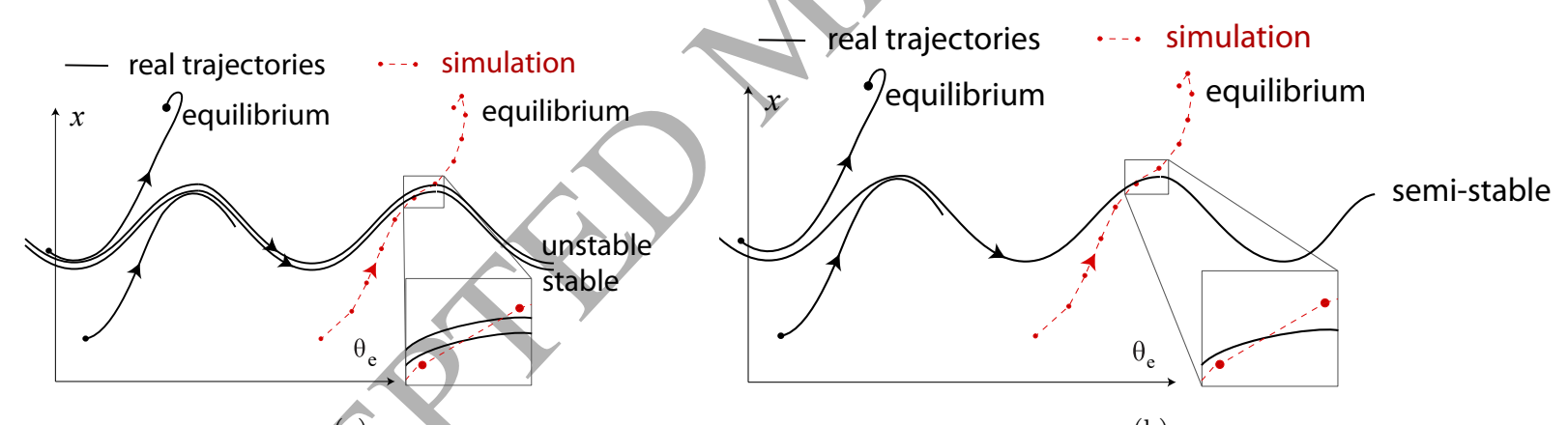

(b)

Figure 14: Phase portrait of PLL-based circuits dynamical model in the signal's phase space: (a) coexistence of stable periodic trajectory (which is a local hidden attractor - locally attracting, closed, and bounded set in cylindrical phase space) and unstable periodic trajectory close to each other; (b) semistable periodic trajectory.

The localization/of hidden oscillation is shown in Fig. 5, Fig. 10, and Fig. 13 (right subfigures). The case corresponds to the close coexisting attractors and the bifurcation of birth of semistable trajectory (semistable cycle) $[18,51-53]$. In this case the numerical methods are limited by the integration errors (see [54, 55]). However, the cycles may merge together and form semi-stable cycle (see Fig. 14b), which is difficult to reveal by the numerical procedure with any integration step.

\section{Conclusion}

The considered examples motivate to apply rigorous analytical methods for the global analysis of PLL-based circuits. As it was noted in [56] stability in simulations may not imply stability of the physical control system and, thus, stronger theoretical understanding is required.

For two-dimensional models of PLL-based circuits with first-order Loop filters, the phase-plane analysis can be effectively applied (see, e.g. [52]). For high-order filters and multi-dimensional models one can apply the corre- 
sponding modifications of classical stability criteria for the nonlinear analysis of control systems in the cylindrical phase space (see, e.g. [4, 57]). However these methods give often only sufficient conditions and rough estimates. Thus, a comprehensive study of PLL-based circuits with high-order filters is a challenging problem.

\section{Acknowledgment}

This work was supported by Russian Science Foundation (project 14-21-00041p) and Saint-Petersburg State University. The authors would like to thank Roland Best (the founder of the Best Engineering Company, Oberwil, Switzerland and the author of the bestseller on PLL-based circuits [13]) and Giovanni Bianchi (Advantest Europe $\mathrm{GmbH}$ ) for valuable discussion on PLL simulation.

\section{References}

[1] F. Gardner, Phaselock techniques, John Wiley \& Sons, New York, 1966.

[2] A. Viterbi, Principles of coherent communications, McGraw-Hill, New York, 1966.

[3] N. Kuznetsov, G. Leonov, M. Yuldashev, R. Yuldashev, Rigorous mathematical definitions of the hold-in and pull-in ranges for phase-locked loops, IFAC-PapersOnLine 48 (11) (2015) 710-713. doi:10.1016/j.ifacol.2015.09.272.

[4] G. Leonov, N. Kuznetsov, M. Yuldashev, R. Yuldashev, Hold-in, pull-in, and lock-in ranges of PLL circuits: rigorous mathematical definitions and limitations of classical theory, IEEE Transactions on Circuits and SystemsI: Regular Papers 62 (10) (2015) 2454-2464. doi:10.1109/TCSI.2015.2476295.

[5] R. Best, N. Kuznetsov, G. Leonov, M. Yuldashev, R. Yuldashev, Tutorial on dynamic analysis of the Costas loop, Annual Reviews in Control 42 (2016) 27-49. doi:10.1016/j.arcontrol.2016.08.003.

[6] J. Stensby, Phase-Locked Loops: Theory and Applications, Phase-locked Loops: Theory and Applications, Taylor \& Francis, 1997.

[7] J. Piqueira, L. Monteiro, Considering second-harmonic terms in the operation of the phase detector for secondorder phase-locked loop, IEEE Transactions On Circuits And Systems-I 50 (6) (2003) 805-809.

[8] N. Margaris, Theory of the Non-Linear Analog Phase Locked Loop, Springer Verlag, New Jersey, 2004.

[9] V. Matrosov, Nonlinear dynamics of phase-locked loop with the second-order filter, Radiophysics and Quantum Electronics 49 (3) (2006) 239-249. doi:10.1007/s11141-006-0057-6.

[10] J. Kudrewicz, S. Wasowicz, Equations of phase-locked loop. Dynamics on circle, torus and cylinder, World Scientific, 2007.

[11] T. Banerjee, B. C. Sarkar, Chaos and bifurcation in a third-order digital phase-locked loop, International Journal of Electronics and Communications (62) (2008) 86-91.

[12] V. Smirnova, A. Proskurnikov, Phase locking, oscillations and cycle slipping in synchronization systems, in: 2016 European Control Conference Proceedings, 2016.

[13] R. Best, Phase-Locked Loops: Design, Simulation and Application, 6th Edition, McGraw-Hill, 2007.

[14] W. Tranter, T. Bose, R. Thamvichai, Basic Simulation Models of Phase Tracking Devices Using MATLAB, Synthesis lectures on communications, Morgan \& Claypool, 2010.

[15] D. Talbot, Frequency Acquisition Techniques for Phase Locked Loops, Wiley-IEEE Press, 2012.

[16] G. Bianchi, N. Kuznetsov, G. Leonov, M. Yuldashev, R. Yuldashev, Limitations of PLL simulation: hidden oscillations in MATLAB and SPICE, International Congress on Ultra Modern Telecommunications and Control Systems and Workshops (ICUMT 2015) 2016-January (2016) 79-84. doi:10.1109/ICUMT.2015.7382409.

[17] M. Blagov, N. Kuznetsov, G. Leonov, M. Yuldashev, R. Yuldashev, Simulation of PLL with impulse signals in MATLAB: Limitations, hidden oscillations, and pull-in range, International Congress on Ultra Modern Telecommunications and Control Systems and Workshops (ICUMT 2015) 2016-January (2016) 85-90. doi:10.1109/ICUMT.2015.7382410. 
[18] G. Leonov, N. Kuznetsov, Hidden attractors in dynamical systems. From hidden oscillations in HilbertKolmogorov, Aizerman, and Kalman problems to hidden chaotic attractors in Chua circuits, International Journal of Bifurcation and Chaos 23 (1), art. no. 1330002. doi:10.1142/S0218127413300024.

[19] G. Leonov, N. Kuznetsov, T. Mokaev, Homoclinic orbits, and self-excited and hidden attractors in a Lorenzlike system describing convective fluid motion, Eur. Phys. J. Special Topics 224 (8) (2015) 1421-1458. doi:10.1140/epjst/e2015-02470-3.

[20] N. Kuznetsov, Hidden attractors in fundamental problems and engineering models. A short survey, Lecture Notes in Electrical Engineering 371 (2016) 13-25, (Plenary lecture at International Conference on Advanced Engineering Theory and Applications 2015). doi:10.1007/978-3-319-27247-4_2.

[21] S. Goldman, Phase-Locked Loops Engineering Handbook for Integrated Circuits, Artech House, 2007.

[22] T. Emura, L. Wang, M. Yamanaka, H. Nakamura, A high-precision positioning servo controller based on phase/frequency detecting technique of two-phase-type PLL, Industrial Electronics, IEEE Transactions on 47 (6) (2000) 1298-1306.

[23] R. Best, N. Kuznetsov, G. Leonov, M. Yuldashev, R. Yuldashev, Simulation of analog Costas loop circuits, International Journal of Automation and Computing 11 (6) (2014) 571-579. doi:10.1007/s11633-014-0846-x.

[24] G. Bianchi, N. Kuznetsov, G. Leonov, S. Seledzhi, M. Yuldashev, R. Yuldashev, Hidden oscillations in SPICE simulation of two-phase Costas loop with non-linear VCO, IFAC-PapersOnLine 49 (14) (2016) 4550. doi:10.1016/j.ifacol.2016.07.973.

[25] S. Schaefer, W. Rosenkranz, Costas-loop based carrier recovery in optical coherent intersatellite communications systems, in: Ultra Modern Telecommunications and Control Systems and Workshops (ICUMT), 2015 th International Congress on, 2015, pp. 30-34.

[26] J. P. Costas, Receiver for communication system, US Patent 3,047,659 (1962).

[27] R. Best, N. Kuznetsov, O. Kuznetsova, G. Leonov, M. Yuldashev, R. Yuldashev, A short survey on nonlinear models of the classic Costas loop: rigorous derivation and limitations of the classic analysis, in: Proceedings of the American Control Conference, IEEE, 2015, pp. 1296-1302, art. num. 7170912, http://arxiv.org/pdf/1505.04288v1.pdf. doi:10.1109/ACC.2015.7170912.

[28] M. Zakrzhevsky, I. Schukin, V. Yevstignejev, Scientific Proc. Riga Technical Univ. Transp. Engin. 6 (2007) 79.

[29] D. Dudkowski, S. Jafari, T. Kapitaniak, N. Kuznetsov, G. Leonov, A. Prasad, Hidden attractors in dynamical systems, Physics Reports 637 (2016) 1-50. doi:10.1016/j.physrep.2016.05.002.

[30] B. Andrievsky, N. Kuznetsoy, G. Leonov, A. Pogromsky, Hidden oscillations in aircraft flight control system with input saturation, IFAC Proceedings Volumes 46 (12) (2013) 75-79. doi:10.3182/20130703-3-FR4039.00026 .

[31] B. Andrievsky, N. Kuznetsov, G. Leonov, S. Seledzhi, Hidden oscillations in stabilization system of flexible launcher with saturating actuators, IFAC Proceedings Volumes 46 (19) (2013) 37-41. doi:10.3182/201309025-DE-2040.00040.

[32] G. Leonov, N. Kuznetsov, M. Kiseleva, E. Solovyeva, A. Zaretskiy, Hidden oscillations in mathematical model of drilling system actuated by induction motor with a wound rotor, Nonlinear Dynamics 77 (1-2) (2014) $277-288$. doi:10.1007/s11071-014-1292-6.

[33] M. Kiseleva, N. Kondratyeva, N. Kuznetsov, G. Leonov, E. Solovyeva, Hidden periodic oscillations in drilling system driven by induction motor, IFAC Proceedings Volumes 47 (19) (2014) 5872-5877. doi:10.3182/201408246-ZA-1003.02449.

[34] M. Kiseleva, N. Kondratyeva, N. Kuznetsov, G. Leonov, Hidden oscillations in drilling systems with salient pole synchronous motor, IFAC-PapersOnLine 48 (11) (2015) 700-705. doi:10.1016/j.ifacol.2015.09.270.

[35] M. Kiseleva, N. Kuznetsov, G. Leonov, Hidden attractors in electromechanical systems with and without equilibria, IFAC-PapersOnLine 49 (14) (2016) 51-55. doi:10.1016/j.ifacol.2016.07.975.

[36] G. Leonov, N. Kuznetsov, V. Vagaitsev, Localization of hidden Chua's attractors, Physics Letters A 375 (23) (2011) 2230-2233. doi:10.1016/j.physleta.2011.04.037. 
[37] G. Leonov, N. Kuznetsov, V. Vagaitsev, Hidden attractor in smooth Chua systems, Physica D: Nonlinear Phenomena 241 (18) (2012) 1482-1486. doi:10.1016/j.physd.2012.05.016.

[38] P. Li, T. Zheng, C. Li, X. Wang, W. Hu, A unique jerk system with hidden chaotic oscillation, Nonlinear Dynamics 86 (1) (2016) 197-203.

[39] Z. Wei, V.-T. Pham, T. Kapitaniak, Z. Wang, Bifurcation analysis and circuit realization for multiple-delayed Wang-Chen system with hidden chaotic attractors, Nonlinear Dynamics 85 (3) (2016) 1635-1650.

[40] H. Jiang, Y. Liu, Z. Wei, L. Zhang, Hidden chaotic attractors in a class of two-dimensional maps, Nonlinear Dynamics 85 (4) (2016) 2719-2727.

[41] V.-T. Pham, C. Volos, S. Jafari, S. Vaidyanathan, T. Kapitaniak, X. Wang, A chaotic system with different families of hidden attractors, International Journal of Bifurcation and Chaos 26 (08) (2016) 1650139.

[42] S. Jafari, V.-T. Pham, S. Golpayegani, M. Moghtadaei, S. Kingni, The relationship between chaotic maps and some chaotic systems with hidden attractors, Int. J. Bifurcat. ChaosAccepted.

[43] M.-F. Danca, Hidden transient chaotic attractors of Rabinovich-Fabrikant system, Nonlinear Dynamics 86 (2) (2016) 1263-1270.

[44] I. Zelinka, Evolutionary identification of hidden chaotic attractors, Engineering Applications of Artificial Intelligence 50 (2016) 159-167.

[45] N. Kuznetsov, G. Leonov, T. Mokaev, S. Seledzhi, Hidden attractor in the Rabinovich system, Chua circuits and PLL, AIP Conference Proceedings 1738 (1), art. num. 210008.

[46] G. Leonov, N. Kuznetsov, M. Yuldahsev, R. Yuldashev, Analytical method for computation of phase-detector characteristic, IEEE Transactions on Circuits and Systems - II: Express Briefs 59 (10) (2012) 633-647. doi:10.1109/TCSII.2012.2213362.

[47] G. Leonov, N. Kuznetsov, M. Yuldashev, R. Yuldashev, Nonlinear dynamical model of Costas loop and an approach to the analysis of its stability in the large, Signal Processing 108 (2015) 124-135. doi:10.1016/j.sigpro.2014.08.033.

[48] N. Kuznetsov, G. Leonov, S. Seledzhi, M. Yuldashev, R. Yuldashev, Elegant analytic computation of phase detector characteristic for non-sinusoidal signals, IFAC-PapersOnLine 48 (11) (2015) 960-963. doi:10.1016/j.ifacol.2015.09.316.

[49] G. Leonov, N. Kuznetsov, M. Yuldashev, R. Yuldashev, Computation of the phase detector characteristic of a QPSK Costas loop, Doklady Mathematics 93 (3) (2016) 348-353. doi:10.1134/S1064562416030236.

[50] M. Kapranov, Locking band for phase-locked loop, Radiofizika (in Russian) 2 (12) (1956) 37-52.

[51] N. A. Gubar', Investigation of a piecewise linear dynamical system with three parameters, J. Appl. Math. Mech. 25 (6) (1961) 1011-1023.

[52] B. Shakhtarin, Study of a piecewise-linear system of phase-locked frequency control, Radiotechnica and electronika (in Russian) (8) (1969) 1415-1424.

[53] L. Belyustina, V. Brykov, K. Kiveleva, V. Shalfeev, On the magnitude of the locking band of a phase-shift automatic frequency control system with a proportionally integrating filter, Radiophysics and Quantum Electronics 13 (4) (1970) 437-440.

[54] M. Biggio, F. Bizzarri, A. Brambilla, G. Carlini, M. Storace, Reliable and efficient phase noise simulation of mixed-mode integer-N phase-locked loops, in: Circuit Theory and Design (ECCTD), 2013 European Conference on, IEEE, 2013, pp. 1-4.

[55] M. Biggio, F. Bizzarri, A. Brambilla, M. Storace, Accurate and efficient PSD computation in mixed-signal circuits: A time-domain approach, Circuits and Systems II: Express Briefs, IEEE Transactions on 61 (11) (2014) 905-909.

[56] T. Lauvdal, R. Murray, T. Fossen, Stabilization of integrator chains in the presence of magnitude and rate saturations: a gain scheduling approach, in: Proc. IEEE Control and Decision Conference, Vol. 4, 1997, pp. 4404-4005.

[57] G. Leonov, N. Kuznetsov, Nonlinear Mathematical Models of Phase-Locked Loops. Stability and Oscillations, Cambridge Scientific Publisher, 2014. 